\title{
Fermi-LAT Gamma-Ray Variability Study of Misaligned AGN
}

\author{
P. Grandi ${ }^{1, a}$, E. Torresi ${ }^{1}$, A. De Rosa ${ }^{1}$, S. Rainó ${ }^{2}$, and G. Malaguti ${ }^{1}$ \\ ${ }^{1}$ INAF-IASFBO via Gobetti 101, I-40129 Bologna, Italy \\ ${ }^{2}$ Istituto Nazionale di Fisica Nucleare, Via E. Orabona 4, I-70125 Bari, Italy
}

\begin{abstract}
We review the gamma-ray variability properties of AGN with a jet not directly pointing at the observer, i.e. Misaligned AGN. This class is mainly populated by nearby low power radio galaxies (i.e. FRIs). The high power radio sources (i.e. FRIIs) are indeed rare in the GeV sky. Our Fermi-LAT inspection of 4 years of data reveals different temporal behaviors, supporting the idea that beaming/jet structural differences could characterize the FRI-FRII jets. The case of 3C111 (FRII) and NGC6251 (FRI) are treated in detail. New insights into the location and dimension of the gamma-ray dissipation regions are provided for galaxies with different radio morphologies.
\end{abstract}

\section{Introduction}

Radio Loud (RL) AGN are active galaxies with giant plasma outflows through which the black hole transfers huge amount of energy, momentum and angular momentum to the ambient. Blazars, i.e. BL LACs (BLs) and Flat Spectrum Radio Quasars (FSRQs), have a jet directly pointing towards the observer, while Misaligned AGN (MAGN), i.e. radio galaxies (RGs) and Steep Spectrum Radio Quasars (SSRQs), are characterized by jets oriented away from the line of sight. On the base of their radio morphology, MAGNs are classified as Fanaroff-Riley I (FRI) or Fanaroff-Riley II (FRII). Twin jet structures are found in FRI radio galaxies, while edge-brightened radio lobes are observed in FRII radio galaxies. FRIIs are bright $\left(\mathrm{P}_{178 \mathrm{MHz}}>10^{25}\right.$ Watt $\left.\mathrm{Hz}^{-1} \mathrm{sr}^{-1}\right)$ and probably host a standard (geometrically thin, and optically thick) accretion disk in their central regions. On the contrary, FRIs are less powerful and have a central engine less efficient in converting gravitational power into radiation (i.e. an Advection-Dominated Accretion Flow)[1]. According to the unification scenario, FRI and FRII radio galaxies represent the parent population of BL and FSRQ, respectively, with SSRQ in between.

The high-energy radiation in RL AGN involves inverse-Compton up-scattering of lower-energy photons by relativistic electrons in the jet. In FRIs (and BLs), the seed photons are produced within the jet (SynctrotronSelf-Compton (SSC) model). In FRII RGs (and FS$\mathrm{RQs} / \mathrm{SSRQs}$ ), the radiation is mainly external (External Compton (EC) process), as the jet propagates through an environment rich in infrared-optical-UV photons.

In Blazars the jet orientation favors the radiation amplification by relativistic Doppler effect. Indeed Blazars are the majority of the extragalactic sources included in the

\footnotetext{
ae-mail: grandi@iasfbo.inaf.it
}

Table 1. MAGN detected after 2 years of Fermi-LAT survey

\begin{tabular}{|c|c|c|}
\hline Source & FR type & References \\
\hline $3 \mathrm{C} 78$ & FRI & {$[2],[4]$} \\
\hline NGC 1275 & FRI & {$[2],[4],[3]$} \\
\hline IC $310^{1}$ & FRI/BL? & [3] \\
\hline $3 \mathrm{C} 111$ & FRII & [2], [4] \\
\hline $3 \mathrm{C} 120$ & $\mathrm{FRI}^{2}$ & [4] \\
\hline FornaxA & FRI & [3] \\
\hline PKS 0625-354 & FRI & [2], [4], [3] \\
\hline $3 \mathrm{C} 207$ & FRII/SSRQ & {$[2],[4],[3]$} \\
\hline PKS 0943-76 & FRII & [2], [4], [3] \\
\hline M 87 & FRI & {$[2],[4],[3]$} \\
\hline Centaurus A & FRI & {$[2],[4],[3]$} \\
\hline Centaurus $\mathrm{B}^{1}$ & FRI & [3] \\
\hline NGC 6251 & FRI & {$[2],[4],[3]$} \\
\hline $3 \mathrm{C} 380$ & FRII/SSRQ & {$[2],[4],[3]$} \\
\hline
\end{tabular}

First (1FGL [2]) and Second Fermi-LAT (2FGL [3]) catalogs. Despite of their disadvantageous geometry, MAGN were also detected by Fermi during the first 2 years of sky survey.

One of most impressive result of the high energy study of MAGN is the disparity of detection between powerful and weak radio sources. As shown in table 1, where all the MAGN detected in 24 months of data are listed, 10 out of 14 sources are classified as FRI radio galaxies ${ }^{1}$. Although longer debated, the question is still open. The most simple approach assumed that the FRIIs have a lower MeV-GeV detection rate because of their location at higher redshift (i.e. they are fainter). However, a study of [5] showed that, if a proportionality between the radio core and the gammaray emission [6] is assumed, FRIIs are bright enough to be visible at high energies. It is then possible that the FRIFRII gamma-ray dichotomy is related to different intrinsic

\footnotetext{
${ }^{1} 3 \mathrm{C} 120$ is a peculiar source. It is classified as FRI but hosts a very efficient accretion disk.
} 
properties. For example [7] noted that if the high energy emission is due to Compton scattering of external photons, the Doppler boosting is stronger and the beaming cone narrower than in the SSC case. Thus a beaming difference could explain the lower gamma-ray detection rate of more powerful radio galaxies. Another possibility is that onezone homogeneous SSC models is oversimplified. The jet could be structured and characterized by different regions at different velocities. In FRI radio galaxies, the presence of slower components could play an important role in amplifying the $\mathrm{GeV}$ emission (see for example[8]). In order to explore this issue from a different point of view, we started a MAGN study of the $\gamma$-ray variability. Here we present preliminary results of this analysis.

\section{MAGN Variability}

Some MAGN, listed in 1FGL paper are not present in the 2FGL catalog (see table 1), denoting that some sources appear above the background only for short periods of time and disappear when long integration times are considered. This is a first indication of the temporal activity of MAGN. In order to explore better this point, we consider the variability index $\left(T S_{\text {var }}\right)$ reported in the 2 FGL catalog. This parameter, based on a likelihood analysis, is used to quantify the variability of a source (for more details see [3]) and refers to light curves of 24 bins of 1 month each. A $T S_{\text {var }}>41.6$ corresponds to a probability that the source is variable at a $99 \%$ confidence level. In figure 1 the variability index is plotted as a function of the $\gamma$-ray flux $\left(F_{>100 \mathrm{MeV}}\right.$ phot $\left.\mathrm{cm}^{-2} \mathrm{~s}^{-1}\right)$ for BLs, FSRQs and MAGN. Three MAGN, two of which are FRII/SSRQs, meet the variability criterium. Interesting enough, the majority of the FRI radio galaxies occupies the plot region below the $T S_{\mathrm{var}}=41.6$ (dotted line).

The inspection of the light curves suggests a different temporal behaviors of the two radio classes. Example of typical FRI and FRII light curves are shown in figure 2. FRIs are, on average, detected for most of the time. Their time coverage, defined as the ratio between the bins with detection and the total number of bins $\left(\mathrm{N}_{d e t} / N_{t o t}\right)$ is around $70 \%$. The FRI light curves do not generally show variability structures. When a standard $\chi^{2}$ test is applied to each light curve, the hypothesis of a constant flux is rejected only in two cases (i.e. NGC6251 and NGC1275). By contrast, FRIIs are only sporadically detected with an average time coverage less than $30 \%$. It seems that FRII sources become observable only when a short and intense flare boosts their flux above the LAT sensitivity threshold (figure 2 - right panel).

To further investigate the time variability properties of MAGN we generated the light curve of the sources in table $1^{2}$ considering 4 years of observation and dividing the total period in 16 time intervals of 3 months duration. For each bin time, the flux was estimated using the likelihood analysis described in [2]. The significance of the source

${ }^{2}$ IC 310 and Centaurus B were not considered because of the complexity of their $\gamma$-ray environment.

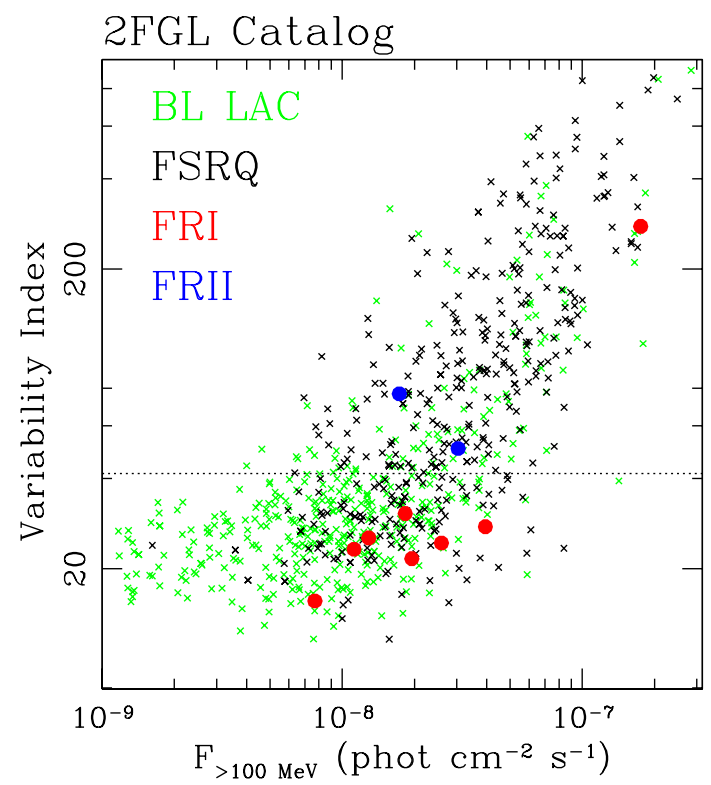

Figure 1. Variability index plotted as a function of the $\gamma$-ray flux for different classes of AGN. Data are from the 2FGL catalog. The horizontal dotted line corresponds to a variability index = 41.6. Above this value the probability that a source is variable is $>99 \%$. AGN with jets pointed away from the observer follow the same trend of the Blazars. As expected, the variability index increases with the flux, as the flux changes are better detected in brighter sources. (Preliminary figure)

was given by the test statistic TS $=2 \Delta \log$ (likelihood) between models with and without the source. The flux in a bin was substituted by a $95 \%$ upper limit when the TS is less than 10 (i.e. when the source detection significance was less than $\sim 3 \sigma)$.

\section{Flaring and Stationary Regions in MAGN}

It is possible that the FRIIs flare events (traced by the light curves) are associated to the ejection of a new blob from the radio core. In this respect, the case of 3C 111 is particularly enlightening. The light curve of 3C 111, limited to the first 2 years of LAT data, shows only one bin detection. The source spends not more than two months flaring and then disappears. This fast variability ( $\Delta t \sim 60$ days) attests the compactness of the $\gamma$-ray emitting region that can not be larger than $R \lesssim \Delta t \delta c \lesssim 0.03 \delta$ pc (where $\delta$ is the beaming factor). It was a fortunate coincidence that a multifrequency campaign of 3C 111 [9] was ongoing during the same period. As shown in figure 3 (left panel), an increase of the mm, optical and X-ray fluxes was observed when the source flared in the $\gamma$-ray band. This was interpreted as a clear indication of a co-spatiality of the events and triggered an accurate analysis of the Very Long Baseline Array (VLBA) jet imaging at $43 \mathrm{GHz}$. As suggested by [9] the multifrequency flare was caused by the passage of a superluminal knot through the jet core. On the basis of the evolution of the 3C 111 jet features, [10] could later estimate a distance of the $\gamma$-ray dissipation region from the black hole not larger than $0.3 \mathrm{pc}$. 

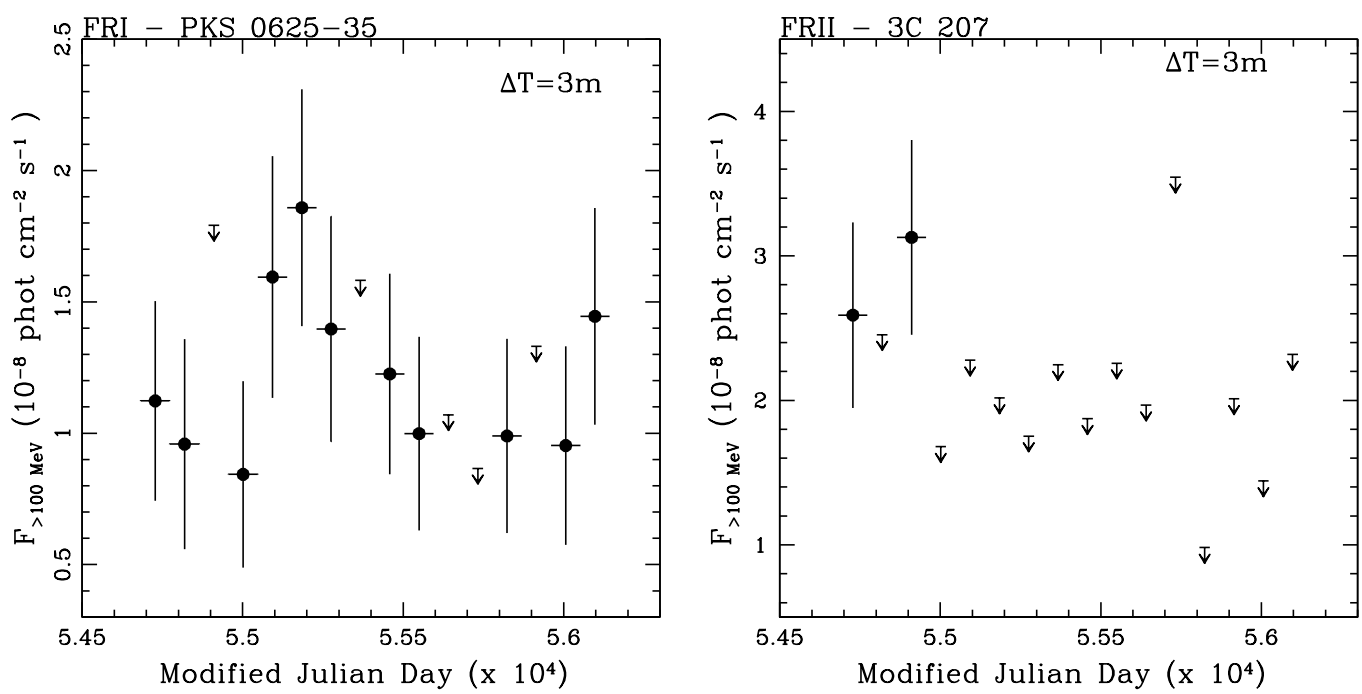

Figure 2. Light curves for the FRI radio galaxy PKS0625-35 (left panel) and the FRII quasar 3C207 (right panel). Each bin corresponds to 3 months of observations in the $100 \mathrm{MeV}-100 \mathrm{GeV}$ band. While PKS0625-35 is detected for $~ 70 \%$ of the time, 3C 207 is observed in only two occasions implying a time coverage of $\mathrm{N}_{\text {det }} / N_{\text {tot }} \sim 6 \%$. (Preliminary figure)
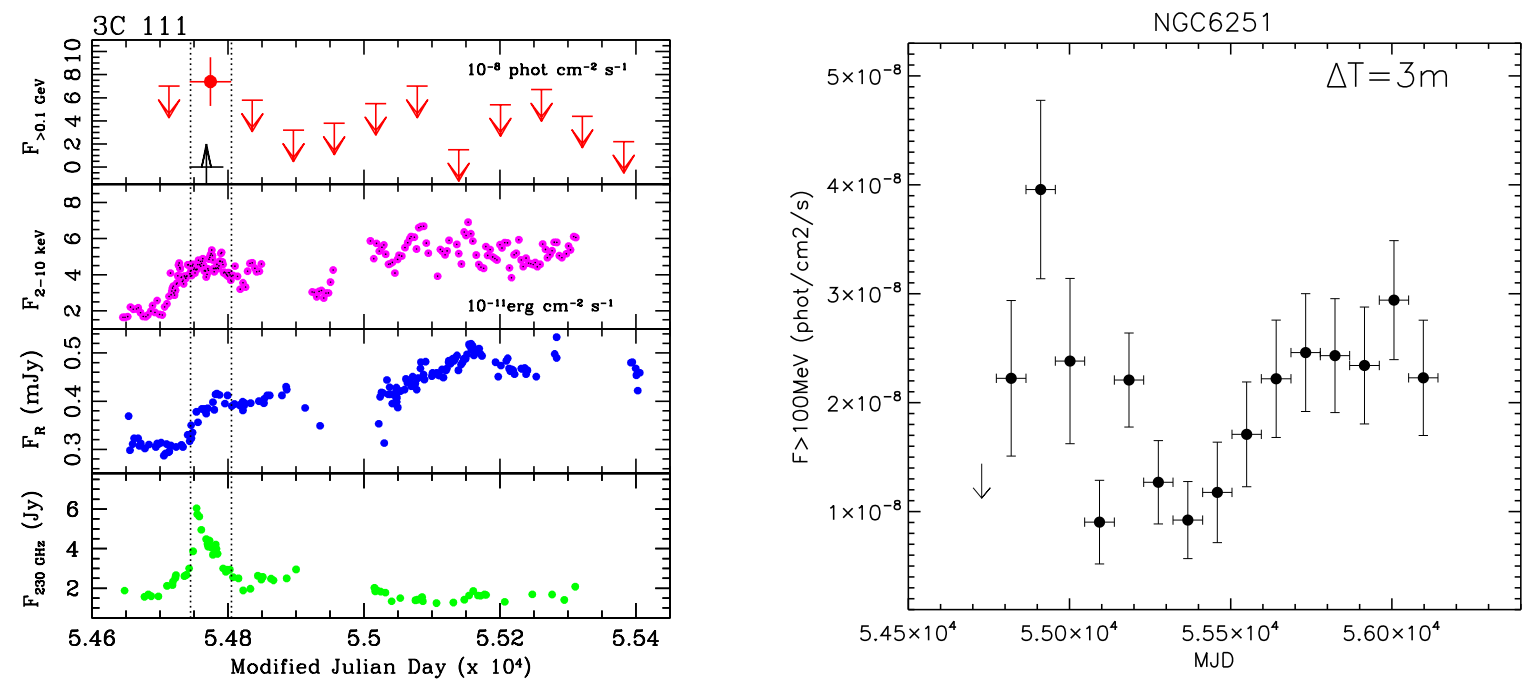

Figure 3. Left panel - Millimeter (230 GHz), optical (R), X-ray (2.4-10 keV) and $\gamma$-ray (100 MeV-100 GeV) light curves of $3 \mathrm{C} 111$. The multifrequency flare is due to the passage of a superluminal knot through the jet core. The black arrow indicates the time when the radio blob was ejected by the core. Right panel - 48 month light curve of NGC6251 in the $100 \mathrm{MeV}-100 \mathrm{GeV}$ band. A $\chi^{2}$ test confirms the source variability. The probability that the source is constant is $P_{\chi^{2}}=2.2 \times 10^{-3}$. (Preliminary figure)

The localization of the $\gamma$-ray emission regions in FRI radio galaxies is more difficult, as they are generally quiescent (or undergo small flux changes not detectable by Fermi). However, the detection of $\mathrm{GeV}$ variability in NGC1275 and NGC6251 (figure 3- right panel) points to the presence of compact $\left(<\mathrm{a}\right.$ few $\left.10^{-2} \mathrm{pc}\right)$ emitting (flaring) regions also in FRIs. Nevertheless the picture could be more complex. As shown by the Centaurus A study, both the jet and the extended radio lobes can be sites of $\gamma$-ray production [11]. Multiple zones (not always resolved by the LAT instrument) could then contribute to the high energy emission (at least) in the closest MAGN (i.e in FRIs). NGC6251, one of the few MAGN with a radio extension larger than the LAT Point Spread Function (PSF), seems to support this possibility. If modeled with only one source, its $\gamma$-ray emission peaks on the jet at $7 \pm 3$ arcminutes $^{3}$ from the radio core (figure 4 - left panel). There are actually some observational evidences that high energy bursts can occur in knots along the jet [12][13], but the $\gamma$-ray flaring region of NGC 6251 would be at least 100 times more distant. A compact variable feature so far from the central engine is difficult to explain within the current models. Another (more plausible) interpretation is that the $\gamma$-rays radiation is due to different contributions. Indeed an

\footnotetext{
${ }^{3} 1$ " corresponds to $485 \mathrm{pc}$, assuming $\mathrm{h}=0.71, \Omega_{m}=0.27, \Omega_{\Lambda}=0.73$, where $\mathrm{H}_{0}=100 h \mathrm{~km}^{-1} \mathrm{~s}^{-1} \mathrm{Mpc}^{-1}$
} 

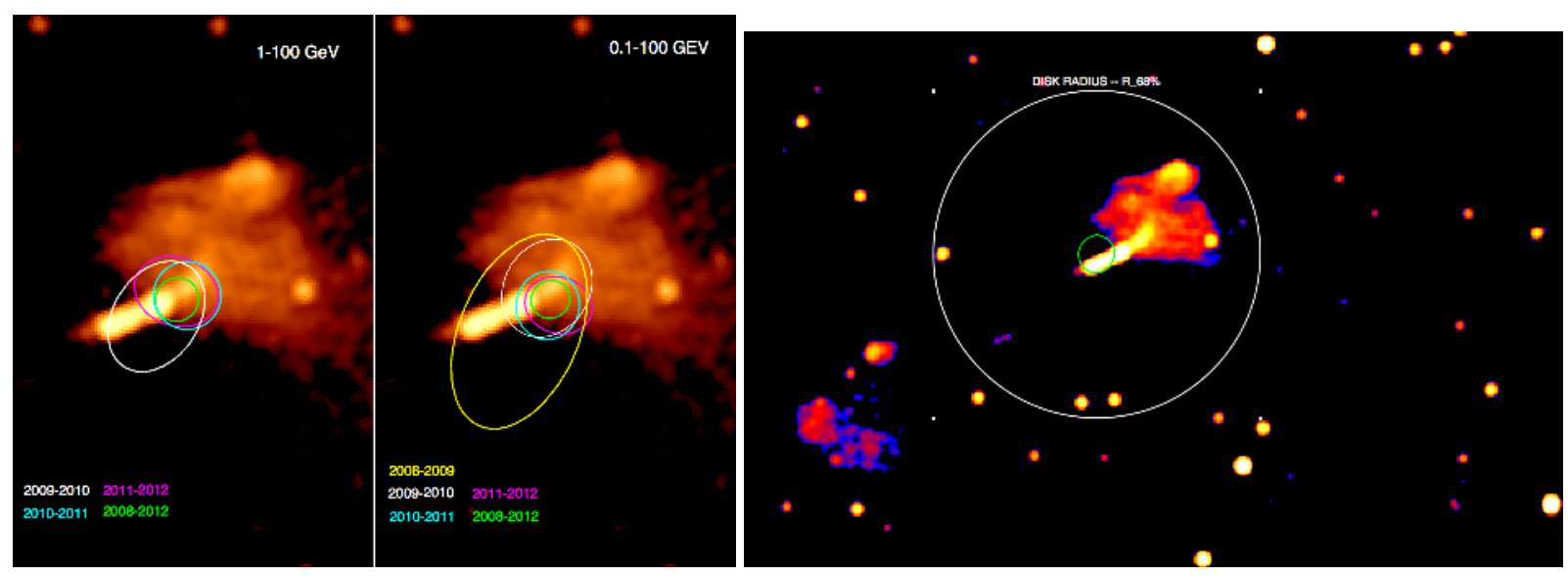

Figure 4. Left panel-NGC 6251 radio map at $92 \mathrm{~cm}$. Ellipses represent the best-fit position (68\% uncertainty region) of the $\gamma$-ray source (assumed pointlike) obtained considering all the LAT photons between 0.1 and $100 \mathrm{GeV}$ (left map) and only the photons $>1$ $\mathrm{GeV}$ (right map). The green ellipse corresponds to an integration time of 48 months, the other curves to a single year of observation (2008-2009: yellow , 2009-2010: white, 2010-2011: cyan, 2011-2012: magenta). In correspondence of the first year of observation, the $68 \%$ uncertainty region in the $1-100 \mathrm{GeV}$ range is not shown. During the first 12 months, the source was in flare and exhibited a very soft spectrum with no significant emission above a few GeV. Right panel - Same radio image at $92 \mathrm{~cm}$ with the extended emission containing $68 \%$ of the $\gamma$-ray intensity (white circle). The green circle represents the best-fit $\gamma$-ray localization region of the disk center ( $1 \sigma$ uncertainty). (Preliminary figures)

extended source with a disk-like shape, including the entire western jet-lobe structure, can model the $\gamma$-ray emission $^{4}$. The significance of the extension of the source is $\sim 3 \sigma$ if the entire LAT energy band is explored, but increases to $4 \sigma$ if the analysis is restricted to the $1-100 \mathrm{GeV}$ photons. Moreover the radio core falls within the $68 \%$ circle constraining the emitting disk center (see figure 4-right panel). It seems then natural to associate the compact and flaring source with the radio core and identify the other dissipation region (probably stationary) with the extended western radio lobe.

\section{Discussion and Conclusions}

The analysis of 4 years of $\gamma$-ray data (from 2008 to 2012) of a sample of 12 MAGN has revealed that the light curves of FRI and FRII sources are different. FRII radio sources are often below the LAT sensitivity threshold with a time coverage less than $30 \%$. On the contrary, the FRI sources are detected, on average, for $\sim 70 \%$ of the time.

Indications are that FRIIs become detectable at MeV$\mathrm{GeV}$ energies during short and intense flares, as suggested by 3C 111. A likely combination of simultaneous mmoptical-X-ray- $\gamma$-ray observations, associated to a VLBA campaign, allowed to relate the gamma-ray detection of this source to the ejection of a new radio blob from the core and to constrain the dimension $(<0.1 \mathrm{pc})$ and the position $(<0.3 \mathrm{pc}$ from the black hole $)$ of the $\gamma$-ray source.

While FRIIs are active and flaring sources, FRIs are generally characterized by structureless light curves. It is possible that this different temporal behavior reflects jet intrinsic differences. Unlike FRIIs, the FRI jet could be

\footnotetext{
${ }^{4}$ Details of the analysis of extended source with Fermi can be found in [14]
}

more stratified/structured with most of $\gamma$-ray radiation produced in less beamed more extended regions (for example, in external layers surrounding an inner fast spine). The jet emission could be also diluted by radiation produced at kpc scales. In NGC6251, the LAT data suggest the presence of an extended $\gamma$-ray region, probably coincident with the western lobe. This is the second FRI radio galaxy, after Centaurus A, showing high energy photon emission outside the jet. For sources with sufficiently bright extended components, the difficulty in detecting the core variability might be partially explained with the presence of lobe emission.

The Fermi LAT Collaboration acknowledges generous ongoing support from a number of agencies and institutes that have supported both the development and the operation of the LAT as well as scientific data analysis. These include the National Aeronautics and Space Administration and the Department of Energy in the United States, the Commissariat à l'Energie Atomique and the Centre National de la Recherche Scientifique / Institut National de Physique Nucléaire et de Physique des Particules in France, the Agenzia Spaziale Italiana and the Istituto Nazionale di Fisica Nucleare in Italy, the Ministry of Education, Culture, Sports, Science and Technology (MEXT), High Energy Accelerator Research Organization (KEK) and Japan Aerospace Exploration Agency (JAXA) in Japan, and the K. A. Wallenberg Foundation, the Swedish Research Council and the Swedish National Space Board in Sweden.

\section{References}

[1] Marchesini, D., Celotti, A., Ferrarese, L. , MNRAS, 351, 733 (2004)

[2] Abdo, A. A., ApJS, 188, 405 (2010) [1FGL]

[3] Nolan, P. L., ApJS, 199, 31 (2012) [2FGL]

[4] Abdo A.A., et al. ApJ, 720, 912 (2010) [MAGN] 
[5] Grandi, P. \& Torresi, E. Fermi \& Jansky Proceeding (2011) (arXiv:1205.1686)

[6] Ackermann, M. et al., ApJ, 741, 30 (2011)

[7] Dermer, C. D. ApJ, 446, L63 (1995)

[8] Ghisellini, G., Tavecchio F., Chiaberge, M., A\&A, 432, 401 (2005)

[9] Chatterjee, R., Marscher, A. P., Jorstad, S. G., et al., ApJ, 734, 43 (2010)
[10] Grandi, P., Torresi, E, Stanghellini, C., ApJ, 751, L3 (2012)

[11] Abdo A. A., et al., Science, 328, 725 (2010)

[12] Agudo, I., Marscher, A. P., Jorstad, S. G., et al. ApJ 735, L10 (2011)

[13] Jorstad, S. G., Marscher, A. P., Schmidt, P. S. et al. ApJ , in press (2013) (arXiv:1307.2522)

[14] Lande, J., Ackermann, A., Allafort, A., et al., ApJ, 756, 5 (2012) 